\title{
The iCub Cognitive Humanoid Robot: An Open-System Research Platform for Enactive Cognition
}

\author{
Giulio Sandini ${ }^{1}$, Giorgio Metta ${ }^{1,2}$, and David Vernon ${ }^{3}$ \\ ${ }^{1}$ Italian Institute of Technology (IIT), Italy \\ ${ }^{2}$ University of Genoa, Italy \\ ${ }^{3}$ Etisalat University College, UAE
}

\begin{abstract}
This paper describes a multi-disciplinary initiative to promote collaborative research in enactive artificial cognitive systems by developing the $i C u b$ : a open-systems 53 degree-of-freedom cognitive humanoid robot. At $94 \mathrm{~cm}$ tall, the $\mathrm{iCub}$ is the same size as a three year-old child. It will be able to crawl on all fours and sit up, its hands will allow dexterous manipulation, and its head and eyes are fully articulated. It has visual, vestibular, auditory, and haptic sensory capabilities. As an open system, the design and documentation of all hardware and software is licensed under the Free Software Foundation GNU licences so that the system can be freely replicated and customized. We begin this paper by outlining the enactive approach to cognition, drawing out the implications for phylogenetic configuration, the necessity for ontogenetic development, and the importance of humanoid embodiment. This is followed by a short discussion of our motivation for adopting an open-systems approach. We proceed to describe the iCub's mechanical and electronic specifications, its software architecture, its cognitive architecture. We conclude by discussing the $i \mathrm{Cub}$ phylogeny, i.e. the robot's intended innate abilities, and an scenario for ontogenesis based on human neo-natal development.
\end{abstract}

\section{Enactive Cognition: Why Create a Cognitive Humanoid Robot?}

Until recently, the study of cognition and the neuro-physiological basis of human behaviour was the subject of quite separate disciplines such as psychology, neurophysiology, cognitive science, computer science, and philosophy, among others. Cognitive processes were mainly studied in the framework of abstract theories, mathematical models, and disembodied artificial intelligence. It has now become clear that cognitive processes are strongly entwined with the physical structure of the body and its interaction with the environment. Intelligence and mental processes are deeply influenced by the structure of the body, by motor abilities and especially skillful manipulation, by the elastic properties of the muscles, and the morphology of the retina and the sensory system. The physical body and its actions together play as much of a role in cognition as do neural processes, and 
human intelligence develops through interaction with objects in the environment and it is shaped profoundly by its interactions with other human beings.

This new view of artificial intelligence represents a shift away from the functionalism and dualism of cognitivism and classical AI towards an alternative position that re-asserts the primacy of embodiment, development, and interaction in a cognitive system [1. Cognitivism and classical physical symbol systems $\mathrm{AI}$ are dualist in the sense that they make a fundamental distinction between the computational processes of the mind and the computational infrastructure of the body, and they are functionalist in the sense that the computational infrastructure is inconsequential: any instantiation that supports the symbolic processing is sufficient. They are also positivist in the sense that they assert a unique and absolute empirically-accessible external reality that is apprehended by the senses and reasoned about by the cognitive processes.

This contrasts with the emergent embodied approach which is based to a greater or lesser extent on principles of self-organization [2/3] and best epitomized by enactive approaches originally formulated in the work of Maturana and Varela [4/5]6/7/2 [8]9. The enactive stance asserts that cognition is the process whereby an autonomous system becomes viable and effective in its environment. In this, there are two complementary processes operating: one being the codetermination of the system and environment (through action and perception and contingent self-organization) and the second being the co-development of the system as it adapts, anticipates, and assimilates new modes of interacting.

Co-determination implies that the cognitive agent is specified by its environment and at the same time that the cognitive process determines what is real or meaningful for the agent. Co-determination means that the agent constructs its reality (its world) as a result of its operation in that world. Perception provides the requisite sensory data to enable effective action [9] but it does so as a consequence of the system's actions. Thus, cognition and perception are functionally-dependent on the richness of the system's action interface [10].

Co-development is the exploratory cognitive process of establishing the possible space of mutually-consistent interaction between the system and its environment. The space of perceptual possibilities is predicated not on an objective environment, but on the space of possible actions that the system can engage in whilst still maintaining the consistency of the coupling with the environment. Through this ontogenetic development - through interaction - the cognitive system develops its own epistemology, i.e. its own system-specific history- and context-dependent knowledge of its world. This knowledge that has meaning exactly because it captures the consistency and invariance that emerges from the dynamic self-organization in the face of environmental coupling.

It is important to understand what exactly we mean here by the term interaction. It is a shared activity in which the actions of each agent influence the actions of the other agents engaged in the same interaction, resulting in a mutually constructed pattern of shared behavior [11. This aspect of mutually constructed patterns of complementary behaviour is also emphasized in Clark's 
notion of joint action [12. According to this definition, explicit meaning is not necessary for anything to be communicated in an interaction: it is simply important that the agents are mutually engaged in a sequence of actions. Meaning then emerges through shared consensual experience mediated by interaction.

Enactive approaches assert that the primary model for cognitive learning is anticipative skill construction rather than knowledge acquisition and that processes that both guide action and improve the capacity to guide action while doing so are taken to be the root capacity for all intelligent systems [13. While cognitivism entails a self-contained abstract model that is disembodied in principle because the physical instantiation of the systems plays no part in the model of cognition [14[15]. In contrast, enactive approaches are intrinsically embodied and the physical instantiation plays a pivotal constitutive role in cognition [14 16 17]. A strong consequence of this is that one cannot short-circuit the ontogenetic development because it is the agent's own experience that defines its cognitive understanding of the world in which it is embedded. Furthermore, since cognition is dependent on the richness of the system's action interface and since the system's understanding of its world is dependent on its history of interaction, a further consequence of enactive $\mathrm{AI}$ is that, if the system is to develop an understanding of the world that is compatible with humans, the system requires a morphology that that is compatible with a human. It is for this reason that a robot which is to be used in the research of human-centred natural and artificial cognition should be humanoid and should possess as rich a set of potential actions as possible.

\section{Why Open-Systems?}

The $i C u b$ is a freely-available open system. This openness is guaranteed by releasing the mechanical and electronic design under a GNU Free Document Licence (FDL) and all embedded software (controller software, interface software, and cognition software) under a GNU General Public Licence. Thus, the scientific community can use it, copy it, and alter it, provided that all alterations to the humanoid design and the embedded software are also made available under a FDL/GPL.

We have two goals in making the $i C u b$ so open. First, we hope that it will become the research platform of choice for the scientific community. This will help establish a de facto standard and therefore increase the likelihood of collaboration among research groups and, consequently, the amount of resources that can be shared among these groups. The nature of the GNU licences helps greatly in this. Second, we hope that by removing the very significant cost of system specification, design, and validation, it will lower the barrier to entry in humanoid research both for people who are expert in humanoid robotics and also for those who simply wish to carry out empirical research in cognitive neuroscience science and developmental psychology. 

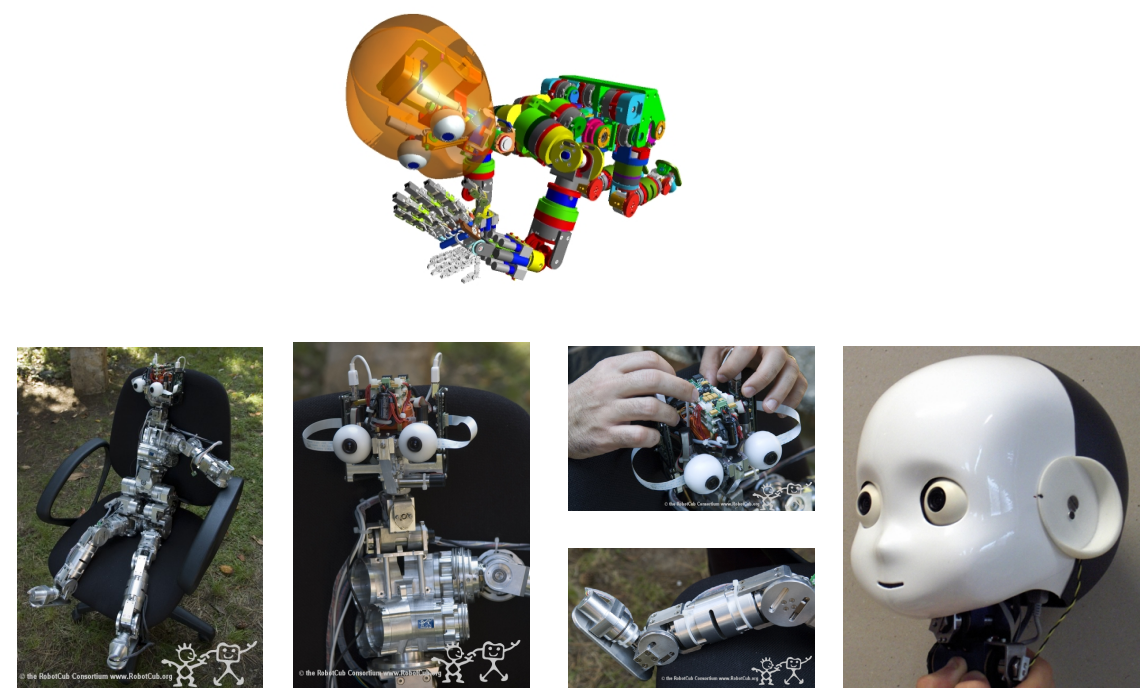

Fig. 1. Details of the $i C u b$ design and construction

\section{The iCub Robot: Mechanical and Electronic Specifications}

To ensure that the iCub's interaction is compatible with humans, for the reasons outlined above, the design is aimed at maximizing the number of degrees of freedom of the upper part of the body, i.e. the head, torso, arms, and hands. The lower body, i.e. the legs and feet, has been designed to support crawling and sitting on the ground in a stable position with smooth autonomous transition from crawling to sitting. The $\mathrm{iCub}$ has 53 degrees of freedom in total: six in the head (two for azimuth \& vergence, one for coupled eye-tilt, and three for the neck) 18, seven degrees of freedom in each of the arms (three in the shoulder, one in the elbow, and three in the wrist), nine degrees of freedom in each of the hands to effect under-actuated control the 17 joints comprising the five fingers), six degrees of freedom in each of the legs (three for the hip joints, one for the knee, and two for the ankle), with the waist also having three degrees of freedom.

The sensory system includes a binocular vision system, touch, audition, and inertial sensors to allow it to coordinate the movement of the eyes and hands, grasp and manipulate lightweight objects of reasonable size and appearance, crawl, and sit up.

Figure 1 shows some details of the current status of design and construction of the $i C u b$.

Although we are focussing for the present on locomotion by crawling, the torque capabilities of the feet, leg, and hip joints have been specified to be sufficient to support bi-pedal locomotion. The development of a bi-pedal gait controller is something we expect will be contributed to the iCub software repository under its GNU licence at some point by a third-party developer. 
All of the motors and sensors are controlled by a suite of DSP chips which channel data over a CAN bus to an on-board PC-104 hub computer. This hub then interfaces over a Gbit ethernet cable to an off-board computer system which takes responsibility for the iCub's high-level behavioural control. Because the $i \mathrm{Cub}$ has so many joints to be configured and such a wealth of sensor data to be processed, to achieve real-time control it is almost inevitable that the $\mathrm{iCub}$ software has be configured to run in parallel on a distributed system of computers. This in turn creates a need for a suite of interface and communications libraries - the $\mathrm{iCub}$ middleware - that will run on this distributed system, effectively hiding the device-specific details of motor controllers and sensors and facilitating inter-process and inter-processor communication. We discuss this middleware briefly in the next section.

\section{The iCub Software Architecture}

We decided to adopt YARP as the iCub middleware [19. YARP (Yet Another Robot Platform) is a multi-platform open-source framework that supports distributed computation with an focus on robot control and efficiency. Yarp comprises a set of libraries which can be embedded in many different systems and robots, and the $i C u b$ is just one of the systems in which YARP is embedded.

YARP provides a set of protocols and a $\mathrm{C}++$ implementation for inter-process communication on a local network (thereby enabling parallel multi-processor computation), for standardization of the hardware interface through run-time dynamically loadable modules, for providing data types for images, vectors, buffers, etc., and for providing various interfaces to commonly used open-source packages (e.g. openCV).

Typically, when writing the iCub software, each module will spawn a set of YARP processes and threads whose complexity will be hidden within the module. The lowest level of the software architecture consists of the level-0 API which provides the basic control of the $\mathrm{iCub}$ hardware by formatting and unformatting IP packets into appropriate classes and data structures. IP packets are sent to the robot via the Gbit Ethernet connection. For software to be compliant to the $i C u b$ the only requirement is to use this and only this API. The API will be provided for both Linux and Windows operating systems. It is then possible to consider multiple levels of software development and level-n APIs that re-use the underlying levels to create even more sophisticated modules. The same rationale of level-0 APIs clearly applies to higher levels.

Higher-level behaviour-oriented application sofware will typically comprise several coarse-grained Yarp processes. This means that to run iCub applications, you only need to invoke each process and instantiate the communication between them. The YARP philosophy is to decouple the process functionality from the specification of the inter-process connections. This encourages modular software with reusable processes that can be used in a variety of configurations that are not dependent on the functionality of the process or embedded code. 
We plan on implementing the $\mathrm{iCub}$ cognitive architecture (see next section) as a set of YARP processes. That is, we expect that each of the iCub phylogenetic abilities as well as the modules for their modulation, for prospection and anticipation, and for self-modification, will be implemented as distinct YARP processes.

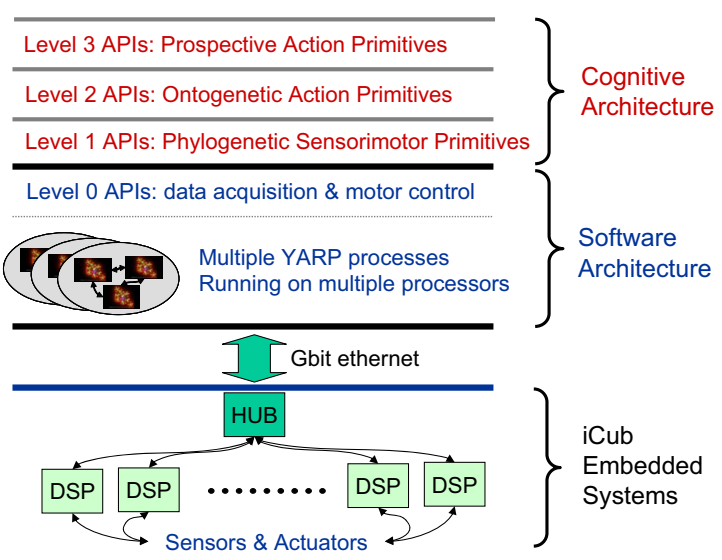

Fig. 2. The layers of the $i C u b$ architecture

\section{The iCub Cognitive Architecture: An Infrastructure for Developmental Learning and Cognition}

The iCub cognitive architecture is based on a survey of cognitivist, emergent, and hybrid cognitive architectures [20, an analysis of the phylogeny and ontogeny of human neonates 21/22, and a review of design principles for developmental systems $23|24| 16]$. The cognitive architecture comprises a network of competing and cooperating distributed multi-functional perceptuo-motor circuits, a modulation circuit which effects homeostatic action selection by disinhibition of the perceptuo-motor circuits, and a system to effect anticipation through perception-action simulation. The modulation circuit comprises three components: auto-associative memory, action selection, and motivation, based loosely on the hippocampus, basal ganglia, and amygdala, respectively, while the anticipatory circuit comprises paired motor-sensor and sensor-motor heteroassociative memories [25]26]27/28|29]30. The anticipatory system allows the cognitive agent to rehearse hypothetical scenarios and in turn to influence the modulation of the network of perception-action circuits. Each perception-action circuit has its own limited representational framework and together they constitute the phylogenetic abilities of the system. The crucial issue of self-modification is catered for in two ways, the first through parameter adjustment of the phylogenetic skills through learning, and the second through the developmental adjustment of the structure and organization of the system so that it is capable 


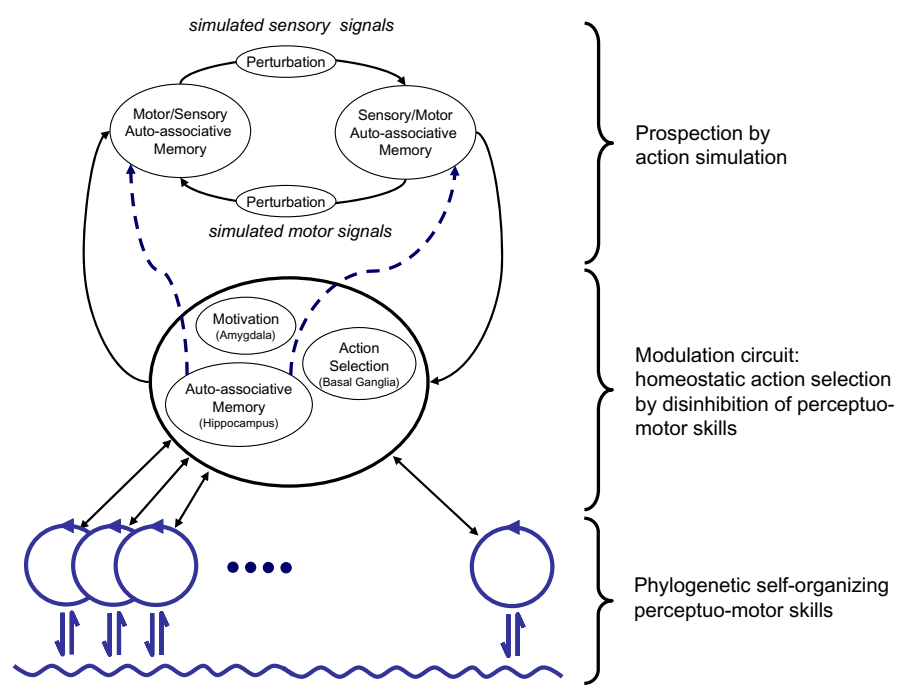

Fig. 3. The $i C u b$ cognitive architecture

of altering its system dynamics based on experience, to expand its repertoire of actions, and thereby adapt to new circumstances. This development, driven by both exploratory and social motives, is effected through the interaction of the anticipatory and modulation circuits, in particular by the update of the long-term anticipatory associative memories by the short-term modulation associative memory. In its current state, this is very much a strawman architecture: it has yet to be validated and it will need to be revised and amended as research progresses. This validation will be both empirical (through experiment) and theoretical (through reference to neuroscientific and psychological models).

\section{The iCub Phylogeny: Innate Abilities}

Development implies the existence of a basis for development; in other words, ontogenesis requires some initial phylogenetic configuration on which to build. This section presents a non-exhaustive list of initially-planned innate perceptuo-motor and cognitive skills that need to be effected in the iCub in order to facilitate its subsequent development. They are organized under the two generic headings perceptuo-motor abilities and enhanced phylogenetic abilities. The perceptuomotor abilities can be considered to be in some sense innate (i.e. operative at or very soon after birth) while the enhanced phylogenetic abilities require some tuning or practice to become effective. These differ from skills that are the result of ontogenesis because there has been little or no modification of the system's state space, i.e. they aren't the result of a process of self-modification or development, but are more akin to learning by on-line parameter estimation. 
The phylogenetic abilities include the ability to distinguish between relative and common motion in the visual field, the ability to ascribe objecthood to parts of the visual field that have persistent and well-defined outer boundaries, the ability to track objects through occlusion, the ability to re-orient gaze towards local perturbations in tactile, auditory, and visual field, and the ability to re-orient and locomote based on local view-dependent landmarks (rather than global scene representations). Since interaction with humans forms an extremely important component of neo-natal development, the phylogentic skills also include a propensity to attend to sounds, movements, and features of the human face and the ability to detect mutual gaze.

The enhanced phylogenetic abilities that we plan to implement initially include a disposition to bring the hand into the visual field, the ability to detect human faces and localize eyes in sensorimotor space, the ability to effect smooth pursuit, and the ability to stabilize percepts in a moving agent, i.e fusion of frames of reference. Subsequently, we will implement abilities concerned with the coordination of perceptuo-motor skills such as ocular modulation of head pose (a tendency whereby head pose is adjusted to centre eye gaze) and the ability to stabilize the percepts arising from moving scenes through successive saccades, i.e. opto-kinetic nystagmus.

We represent this collection of innate phylogenetic abilities in the $\mathrm{iCub}$ cognitive architecture as a series of arrow circles, in the spirit of Maturana and Varela's ideogram of a self-organizing (autopoietic) system 9]; see Figure 3 ,

Note that this is just a partial list of both perceptuo-motor and enhanced phylogenetic abilities. Neonates have other innate skills that we also intend to implement; see [31] for details.

\section{The iCub Ontongeny: A Scenario for Development}

The primary focus of the early stages of ontogenesis of the $i C u b$ is to develop manipulative action based on visuo-motor mapping, learning to decouple motor synergies (e.g. grasping and reaching) 32 33], anticipation of goal states, learning affordances, interaction with other agents through social motives [34|35|36/37] and imitative learning [38|39|40]. Needless to say, ontogenesis and development are progressive. We emphasize the early phases of development, building on the enhanced phylogenetic skills outlined in the previous section and scaffolding the cognitive abilities of the iCub to achieve greater prospection and increased (action-dependent) understanding of its environment and to establish a mutual understanding with other cognitive agents 1

It is important to emphasize that the ontogenetic training program that facilitates the development of the $\mathrm{iCub}$ is biologically inspired and tries to be as faithful as possible to the ontogenesis of neonates. Consequently, the development of manipulative action will build primarily on visuo-motor mapping.

\footnotetext{
${ }^{1}$ An archive of $i C u b$ publications can be found at

www.robotcub.org/index.php/robotcub/more_information/papers.
} 
Once the $\mathrm{iCub}$ has mastered these skills, we will move on to experimental scenarios in which the $\mathrm{iCub}$ learns to develop object manipulation by playing on its own and or with another animate agent, that is, grasping objects and doing things in order to attain effects, like inserting objects into holes, building towers out of blocks etc. At this stage, social learning of object affordances becomes crucial. These scenarios will focus on the use of more than one object, emphasising the dynamic and static spatial relationships between them. In order of complexity, examples include learning to arrange block on a flat-surface, to stack blocks of similar size and shape, to stack blocks on similar shape but different size, and to stack blocks of different shape and size.

The chief point about these scenarios is that they represent an opportunity for the iCub to develop a sense of spatial arrangment (both between itself and objects and between objects), and to arrange and order its local environment in some way. These scenarios also require that the $i \mathrm{Cub}$ learns a set of primitive actions as well as their combination.

\section{Summary}

Enactive embodied emergent cognition represents a fundamental attempt to break with dualist, functionalist, positivist foundations of classical cognitivist AI and to put in place instead a research programme grounded in phenomenology, reasserting the primacy of embodied developmental interaction in cognitive systems. We hope to contribute to this programme by making the iCub cognitive humanoid robot freely available to the research community as cost-effectively as possible and by providing researchers with a suite of cognition software modules for both phylogenetic and ontogenetic functionality. We expect and intend that the research community will improve and add to the iCub hardware and software, sharing their designs and code on the iCub repository at www.icub.org. By working together on this programme, we believe we have a better chance of making the breakthrough in understanding natural and artificial cognition that has eluded classical AI over the past 50 years.

\section{Acknowledgements}

The content of this paper represents the work of many people. These include: Paul Fitzpatrick, Lorenzo Natale, Francesco Nori, Francesco Orabona, Matteo Brunettini, University of Genoa; Paolo Dario, Cecilia Laschi, Anna Maria Carrozza, Giovanni Stellin, Scuola S. Anna, Pisa; Rolf Pfeifer, Gabriel Gomez, Alexandre Schmitz, Yvonne Gustain, Jonas Ruesch, University of Zurich; Claes von Hofsten, Kerstin Rosander, Olga Kochukova, Helena Gronqvist, University of Uppsala; Luciano Fadiga, Laila Craighero, Andrey Olyniyck, Livio Finos, Giovanni Ottoboni, University of Ferrara; Kerstin Dautenhahn, Chrystopher Nehaniv, Assif Mirza, Hatice Kose-Bagci, University of Hertfordshire; José SantosVictor, Alex Bernardino, Luis Montesano, Julio Gomes, Rodrigo Venturo, IST Lisbon; Darwin Caldwell, John Gray, Nick Tsagarakis, University of Salford; 
Aude Billard, Auke Ijspeert, Ludovic Righetti, Sarah Degallier, Ecole Polytechnique Federal de Lausanne; Francesco Becchi, Telerobot S.r.l. Their contributions are gratefully acknowledged.

This work is funded by the European Commission, Project IST-004370 RobotCub, under Strategic Objective 2.3.2.4: Cognitive Systems.

\section{References}

1. Freeman, W.J., Núñez, R.: Restoring to cognition the forgotten primacy of action, intention and emotion. Journal of Consciousness Studies 6(11-12), ix-xix (1999)

2. Varela, F.J.: Whence perceptual meaning? A cartography of current ideas. In: Varela, F.J., Dupuy, J.-P. (eds.) Understanding Origins - Contemporary Views on the Origin of Life, Mind and Society. Boston Studies in the Philosophy of Science, pp. 235-263. Kluwer Academic Publishers, Dordrecht (1992)

3. Clark, A.: Mindware - An Introduction to the Philosophy of Cognitive Science. Oxford University Press, New York (2001)

4. Maturana, H.: Biology of cognition. Research Report BCL 9.0, University of Illinois, Urbana, Illinois (1970)

5. Maturana, H.: The organization of the living: a theory of the living organization. Int. Journal of Man-Machine Studies 7(3), 313-332 (1975)

6. Maturana, H.R., Varela, F.J.: Autopoiesis and Cognition - The Realization of the Living. Boston Studies on the Philosophy of Science. D. Reidel Publishing Company, Dordrecht, Holland (1980)

7. Varela, F.: Principles of Biological Autonomy. Elsevier, North Holland, New York (1979)

8. Winograd, T., Flores, F.: Understanding Computers and Cognition - A New Foundation for Design. Addison-Wesley Publishing Company, Inc., Reading, Massachusetts (1986)

9. Maturana, H., Varela, F.: The Tree of Knowledge - The Biological Roots of Human Understanding. New Science Library, Boston \& London (1987)

10. Granlund, G.H.: The complexity of vision. Signal Processing 74, 101-126 (1999)

11. Ogden, B., Dautenhahn, K., Stribling, P.: Interactional structure applied to the identification and generation of visual interactive behaviour: Robots that (usually) follow the rules. In: Wachsmuth, I., Sowa, T. (eds.) GW 2001. LNCS (LNAI), vol. 2298, pp. 254-268. Springer, Heidelberg (2002)

12. Clark, H.H.: Managing problems in speaking. Speech Communication 15, 243-250 (1994)

13. Christensen, W.D., Hooker, C.A.: An interactivist-constructivist approach to intelligence: self-directed anticipative learning. Philosophical Psychology 13(1), 5-45 (2000)

14. Vernon, D.: The space of cognitive vision. In: Christensen, H.I., Nagel, H.-H. (eds.) Cognitive Vision Systems: Sampling the Spectrum of Approaches. LNCS, pp. 7-26. Springer, Heidelberg (2006)

15. Vernon, D.: Cognitive vision: The case for embodied perception. Image and Vision Computing, 1-14 (in press, 2007)

16. Krichmar, J.L., Edelman, G.M.: Principles underlying the construction of brainbased devices. In: Kovacs, T., Marshall, J.A.R. (eds.) Proceedings of AISB 2006 Adaptation in Artificial and Biological Systems. Symposium on Grand Challenge 5: Architecture of Brain and Mind, Bristol, vol. 2, pp. 37-42. University of Bristol (2006) 
17. Gardner, H.: Multiple Intelligences: The Theory in Practice. Basic Books, New York (1993)

18. Beira, R., Lopes, M., Praça, M., Santos-Victor, J., Bernardino, A., Metta, G., Becchi, F., Saltarén, R.: Design of the robot-cub (icub) head. In: International Conference on Robotics and Automation, ICRA, Orlando (May 2006)

19. Metta, G., Fitzpatrick, P., Natale, L.: Yarp: yet another robot platform. International Journal on Advanced Robotics Systems 3(1), 43-48 (2006)

20. Vernon, D., Metta, G., Sandini, G.: A survey of artificial cognitive systems: Implications for the autonomous development of mental capabilities in computational agents. IEEE Transaction on Evolutionary Computation 11(1), 1-31 (2006)

21. von Hofsten, C.: On the development of perception and action. In: Valsiner, J., Connolly, K.J. (eds.) Handbook of Developmental Psychology, pp. 114-140. Sage, London (2003)

22. von Hofsten, C.: An action perspective on motor development. Trends in Cognitive Science 8, 266-272 (2004)

23. Krichmar, J.L., Edelman, G.M.: Brain-based devices for the study of nervous systems and the development of intelligent machines. Artificial Life 11, 63-77 (2005)

24. Krichmar, J.L., Reeke, G.N.: The darwin brain-based automata: Synthetic neural models and real-world devices. In: Reeke, G.N., Poznanski, R.R., Lindsay, K.A., Rosenberg, J.R., Sporns, O. (eds.) Modelling in the neurosciences: from biological systems to neuromimetic robotics, pp. 613-638. Taylor \& Francis, Boca Raton (2005)

25. Shanahan, M.P.: A cognitive architecture that combines internal simulation with a global workspace. Consciousness and Cognition (to appear)

26. Shanahan, M.P., Baars, B.: Applying global workspace theory to the frame problem. Cognition 98(2), 157-176 (2005)

27. Shanahan, M.P.: Emotion, and imagination: A brain-inspired architecture for cognitive robotics. In: Proceedings AISB 2005 Symposium on Next Generation Approaches to Machine Consciousness, pp. 26-35 (2005)

28. Shanahan, M.P.: Cognition, action selection, and inner rehearsal. In: Proceedings IJCAI Workshop on Modelling Natural Action Selection, pp. 92-99 (2005)

29. Baars, B.J.: A Cognitive Theory of Consciousness. Cambridge University Press, Cambridge (1998)

30. Baars, B.J.: The conscious assess hypothesis: origins and recent evidence. Trends in Cognitive Science 6(1), 47-52 (2002)

31. von Hofsten, C., et al.: A roadmap for the development of cognitive capabilities in humanoid robots. Deliverable D2.1, Project IST-004370 RobotCub (2006), http://www.robotcub.org/index.php/robotcub/more_information/deliverables/

32. Gomez, G., Hernandez, A., Eggenberger-Hotz, P., Pfeifer, R.: An adaptive learning mechanism for teaching a robot to grasp. In: AMAM 2005. International Symposium on Adaptive Motion of Animals and Machines (1999)

33. Natale, L., Metta, G., Sandini, G.: A developmental approach to grasping. In: Developmental Robotics. A 2005 AAAI Spring Symposium, Stanford University, Stanford, CA, USA (2005)

34. Dautenhahn, K., Billard, A.: Studying robot social cognition within a developmental psychology framework. In: Proceedings of Eurobot 1999: Third European Workshop on Advanced Mobile Robots, Switzerland, pp. 187-194 (1999)

35. Dautenhahn, K., Ogden, B., Quick, T.: From embodied to socially embedded agents - implications for interaction-aware robots. Cognitive Systems Research 3(3), 397$428(2002)$ 
36. Blow, M., Dautenhahn, K., Appleby, A., Nehaniv, C.L., Lee, D.: Perception of robot smiles and dimensions for human-robot interaction design. In: RO-MAN 2006. Proceedings of the 15th IEEE International Symposium on Robot and Human Interactive Communication, Hatfield, UK, 6-8 September 2006, pp. 469-474. IEEE Computer Society Press, Los Alamitos (2006)

37. Blow, M., Dautenhahn, K., Appleby, A., Nehaniv, C.L., Lee, D.: The art of designing robot faces-dimensions for human-robot interaction. In: HRI 2006. ACM Proceedings of 1st Annual Conference on Human Robot Interaction, Salt Lake City, Utah, USA, 2-3 March 2006, pp. 331-332. ACM Press, New York (2006)

38. Lopes, M., Santos-Victor, J.: Self-organization in a perceptual network. IEEE Transactions on System Man and Cybernetics - Part B: Cybernetics (June 2005)

39. Hersch, M., Billard, A.: A biologically-inspired model of reaching movements. In: Proceedings of the 2006 IEEE/RAS-EMBS International Conference on Biomedical Robotics and Biomechatronics, Pisa (2006)

40. Hersch, M., Billard, A.: A model for imitating human reaching movements. In: Proceedings of the Human Robot Interaction Conference, Salt Lake City (2006) 